\title{
Implicit assumptions and interpretation bias in youth with severe, chronic social phobia
}

\author{
Lisa Krömer ${ }^{1}(1) \cdot$ Tomasz A. Jarczok $^{1}$ (1) - Heike Althen ${ }^{1} \cdot$ Andreas M. Mühlherr $^{1}$ • Vanessa Howland ${ }^{1,2}$. \\ Stefanie M. Jungmann ${ }^{1,3}$ (D) Christine M. Freitag ${ }^{1}$ (D)
}

Received: 1 July 2020 / Accepted: 16 September 2021 / Published online: 1 October 2021

(c) The Author(s) 2021

\begin{abstract}
Interpretation bias and dysfunctional social assumptions are proposed to play a pivotal role in the development and maintenance of social phobia (SP), especially in youth. In this study, we aimed to investigate disorder-specific implicit assumptions of rejection and implicit interpretation bias in youth with severe, chronic SP and healthy controls (CG). Twenty-seven youth with SP in inpatient/day-care treatment $(M$ age $=15.6$ years, $74 \%$ female $)$ and 24 healthy controls $(M$ age $=15.7$ years, $54 \%$ female) were included. The Implicit Association Test (IAT) and the Affect Misattribution Procedure (AMP) were completed to assess implicit assumptions and interpretation bias related to the processing of social and affective stimuli. No group differences were observed for the IAT controlling for depressive symptoms in the analyses. However, group differences were found regarding interpretation bias $\left(p=.017, \eta_{p}^{2}=.137\right)$. Correlations between implicit scores and explicit questionnaire results were medium to large in the SP group $\left(r=1.28 \mid\right.$ to $\left.|.54|, p_{\text {all }} \leq .05\right)$, but lower in the control group $(r=|.04|$ to $|.46|$, $\left.p_{\text {all }} \leq .05\right)$. Our results confirm the finding of an interpretation bias in youth SP, especially regarding the implicit processing of faces, whereas implicit dysfunctional social assumptions of being rejected do not seem to be specific for SP. Future research should investigate the causal relationship of assumptions/interpretation bias and SP.
\end{abstract}

Keywords Affective Misattribution Procedure · Dysfunctional social assumptions · Implicit Association Test · Implicit Processing $\cdot$ Interpretation Bias $\cdot$ Social Phobia

\section{Introduction}

Anxiety disorders are the most common mental disorders in children and youth [1]. In particular, social phobia (SP) frequently occurs in youth with a prevalence of up to $10 \%$

Stefanie M. Jungmann and Christine M. Freitag shared senior authorship.

Lisa Krömer

lisa.kroemer@kgu.de

Tomasz A. Jarczok

Tomasz.Jarczok@kgu.de

Heike Althen

heike.althen@kgu.de

Andreas M. Mühlherr

andreasmichael.muehlherr@kgu.de

Vanessa Howland

vanessa.howland@uni-greifswald.de

Stefanie M. Jungmann

jungmann@uni-mainz.de
[2-5]. According to the International Classification of Diseases and Related Health Problems (10th edition, ICD-10 [6]), SP describes the fear of scrutiny by other people leading to the avoidance of social situations and is associated with the fear of criticism and low self-esteem. SP has a strong impact on social integration, schooling and vocational

Christine M. Freitag

c.Freitag@em.uni.frankfurt.de

1 Department of Child and Adolescent Psychiatry, Psychosomatics and Psychotherapy, University Hospital Frankfurt, Goethe University, 60528 Frankfurt am Main, Germany

2 Department of Neonatology and Pediatric Intensive Care, University Medicine Greifswald, 17475 Greifswald, Germany

3 Department of Clinical Psychology, Psychotherapy and Experimental Psychopathology, Johannes Gutenberg-University Mainz, 55122 Mainz, Germany 
training and often persists into adulthood if left untreated [7-9].

Youth is a crucial phase for brain development and many cognitive and social changes occur [10]. The reliance on peers is getting more important, leading to increased autonomy from the family of origin, but also coming along with enhanced vulnerability for the development of social anxiety [10]. The importance of this developmental phase is underlined by a peak of social phobia onset in early youth [10]. Several aetiological models have been put forward to explain the occurrence and maintenance of SP [10]. The Cognitive Model of Social Anxiety Disorder has been used as basis for SP specific behavioural intervention approaches [11]. The model assumes, that in an individual with SP, a social situation will trigger assumptions and beliefs about the individual's performance and behaviour. If these assumptions are negative, this will lead to the perception of the respective social situation as being dangerous, resulting in negative automatic thoughts and concerns that one's standards of performance may not be achieved [11]. As a result, negative expectations towards further social situations are accumulating, leading to even more negative assumptions and cognitive biases, such as an interpretation bias [10]. The Cognitive Model of Social Anxiety Disorder has also been suggested to be applicable to youth, taking possible youth-specific factors into account, such as the role of social media, parental factors and peer victimisation [10].

Our study focusses on two crucial elements of the maintenance of SP: negative cognitive assumptions and interpretation bias. In patients with SP, negative assumptions are likely to evolve from firm beliefs about social integration and behaviour, which often include social rejection [10]. Against this background, social situations trigger biased interpretations, e.g. facial expressions are more likely to be interpreted as displaying negative instead of neutral or positive emotions [12]. In line with the Cognitive Model of Social Anxiety Disorders [11], negative cognitive assumptions and interpretation bias are assumed to be associated positively. To date, only a few studies have examined cognitive assumptions in SP. In adults, more negative social assumptions have been shown for highly socially anxious individuals than for low socially anxious participants [13]. In youths with social anxiety, similar results have also been found; one study in the general population [14] found that highly socially anxious youth, aged 14-20 years, showed more dysfunctional social assumptions than low socially anxious youth. Dysfunctional social assumptions have also been shown to predict the severity of social anxiety beyond depression and sociodemographic variables [14]. These negative assumptions about oneself can lead to the interpretation of ambiguous social cues as threatening and socially rejecting [15]. The "tendency to assign a threatening meaning to an objectively ambiguous stimulus with several possible interpretations" is called interpretation bias [16]. The interpretation bias has been studied widely in adults with SP [17-19], but only a few studies have included youth. Highly socially anxious adults have been consistently shown to interpret ambiguous social situations (e.g. receiving no answer to a birthday invitation) more negatively than less socially anxious adults [20, $21]$; in youth, similar results have also been found [22]. For example, highly socially anxious youth aged $12-16$ years, compared to non-socially anxious individuals, interpreted ambiguous social situations (e.g. no definite confirmation of an invitation) more negatively [22]; in another study, a similar group aged 12-17 years, interpreted ambiguous social scenes with a photograph of themselves inserted as the protagonist more negatively than low socially anxious individuals [23]. For adults, a misinterpretation was found not only for neutral-ambiguous stimuli, but also for negative social stimuli. One study found that young adults with higher social anxiety interpreted subtle negative emotional facial expressions more strongly in terms of a negative emotion and as threatening than adults with low social anxiety [24]. In addition, for positive social events, it was found that socially anxious adults endorsed more negative interpretations than patients with other anxiety disorders [25]. When taking a closer look at the interpretation of facial expressions in adults, only a few studies exist which present heterogeneous results. On the one hand, one study did not find differences in the interpretation accuracy of facial expressions [26], while, on the other hand, socially anxious adults were found to be more accurate at identifying facial expressions than healthy controls [27]. Furthermore, socially anxious adults were found to misinterpret expressions, e.g. misinterpreting faces showing disgust as expressing contempt [28], an emotion that is closely related to experiencing rejection [29]. A study on socially anxious children (aged 8-12 years) found no tendency to interpret neutral or positive facial expressions more negatively than healthy controls [12]. Since youth is the age-of-onset for SP, it should be of increasing interest to take a closer look at the interpretation of facial expressions during this crucial developmental phase.

Previous studies on dysfunctional assumptions and negative interpretation bias in SP were mostly based on explicit self-report measures or questionnaires [22]. Dual-processing models, however, underline the importance of two information processing modes: the explicit and the implicit information processing mode [30-32]. The explicit mode is described as a reflective, conscious or effortful information processing, whereas the implicit mode is defined as a more automatic, quick or unconscious processing [30, 33]. Implicit processing was shown to be of importance in many forms of psychopathology [34] and is likely to play a particularly relevant role in youth SP [35]. Youth with SP experience a high level of self-presentational concerns and fear of others' evaluations; this may cause inaccurate or 
incomplete answers on self-reports [36]. Implicit measures are less susceptible to response biases (e.g. social desirability or response tendencies to, for example, medium responses) due to concealing the intention of the measurement and they also do not depend on introspective abilities [33]. These measures are based on behavioural data (e.g. reaction times of categorisations as in the Implicit Association Test, IAT [37]) or on ratings that do not relate to the characteristic of interest (e.g. Chinese characters as in the Affect Misattribution Procedure, AMP [38]). The IAT is proposed to assess associations between a category (e.g. self) and attribute (e.g. rejection), from which conclusions on a person's assumptions, beliefs or schemas can be drawn [39]. The IAT was shown to have the best validity and reliability of seven different implicit self-attribution measures [40]. The AMP assesses automatically activated responses based on a misattribution a person makes about the source of its affect or cognition [41], relying on the fact that people have difficulties disentangling their affective responses when two events occur shortly after each other [42]. The AMP has been used to assess implicit biases in earlier studies on adults with psychiatric disorders $[43,44]$. For the AMP, construct validity and predictive validity with explicit attitude measures were shown [42]. Since implicit assumptions and automatic retrieved implicit biases are proposed to play a relevant role in SP and as both measures have been studied in the context of SP [45, 46], IAT and AMP seem to be the most suitable measuring instruments for the present study's endeavours.

With regard to implicit biases, it has been shown that patients with hypochondriasis show significantly more negative affective reactions in illness prime trials than controls, concluding that negative affective evaluation bias is a unique feature of hypochondriasis [43]. In addition, implicit bias has been studied in patients with eating disorders; these patients showed a higher negative implicit bias for highcalorie food than the healthy controls [44].

Concerning assumptions in SP, the assumptions of being rejected may be of particular importance for SP. Interpersonal rejection sensitivity was found to be not only a specific aspect of depression, but also of SP [47]. In adults with social anxiety disorder, higher rates of explicit interpersonal rejection sensitivity were found compared to healthy controls, independent of self-reported depression [47]. Regarding the implicit assumption of being rejected, only one single study in adults compared SP with healthy controls. Adults with SP and comorbid depression implicitly considered themselves as more socially rejected compared to the SP and control groups; however, the latter two groups did not differ [46]. To our knowledge, no studies exist concerning implicit assumptions of being rejected in youth. A study in youth outpatients with SP (aged 14-20 years) which used the AMP [38] and the IAT (e.g. self-referent words as prime stimuli for assessing implicit self-esteem) [45] to focus on implicit self-esteem did not observe any group differences to the controls. Since explicit assumptions of rejection were found to play a role in SP and implicit assumptions have so rarely been studied, it seems to be of particular importance to examine this further, especially in the vulnerable developmental phase of youth.

Thus, the aim of the current study was to investigate dysfunctional implicit social assumptions and implicit interpretation bias regarding social and emotional stimuli in youth with severe SP in inpatient/day-care hospital treatment. In the present study, a specific version of the AMP to study interpretation bias in reaction to emotional facial expression and a version of the IAT testing implicit assumptions regarding social rejection were implemented. We first hypothesised that youth with SP show stronger implicit assumptions about oneself of being socially rejected than healthy controls. In addition, these implicit assumptions of being rejected are hypothesised to be positively correlated with the explicitly assessed severity of anxiety and SP symptoms. In line with the findings on explicit interpretation bias in adults with SP, we hypothesised that youth with SP, compared to healthy controls, show a stronger implicit interpretation bias, resulting in a more negative interpretation of emotional faces. The resulting pleasantness, experienced when confronted with the facial expressions, is hypothesised to be negatively correlated with the explicitly assessed severity of anxiety and SP symptoms.

\section{Methods}

\section{Participants}

The participants of the SP group (SPG) were recruited at the Department of Child and Adolescent Psychiatry, Psychosomatics and Psychotherapy, University Hospital Frankfurt, Goethe University, while the participants of the control group (CG) were recruited from the general population via social media and advertisements. All participants were aged between 12 and 17 years. Exclusion criteria were an IQ $<70$, uncorrected vision (computer-based tasks) and language proficiency in Chinese/Mandarin (due to the AMP using Chinese characters). IQ was assessed by calculating the mean score of the Vocabulary and Matrix Reasoning subtests of the Wechsler Intelligence Test for Children (WISC-IV) [48] or the Wechsler Intelligence Test for Adults (WAIS-IV;> 16; 11 years) for each participant [49]. Additional exclusion criteria of the SPG were a diagnosis of obsessive-compulsive disorder, tic disorder, acute post-traumatic stress disorder, conduct disorder, bipolar disorder, autism spectrum disorder, substance use disorder, psychotic symptoms and disorders and suicidal ideation. The CG was assessed for the absence 
of psychiatric symptoms by the Youth Self Report [50] and the Child Behavior Checklist [51]. Controls were excluded when at least one subscale of one of the questionnaires indicated clinically relevant symptoms.

The SPG included 27 youth $(M$ age $=15.58$ years, $\mathrm{SD}=1.29$ years, $74 \%$ female) with a diagnosis of social phobia according to the ICD-10 [6]. The youth were seeking inpatient or day-care hospital treatment due to school absenteeism in connection with their psychopathology. Diagnoses and information on school absenteeism were provided by experienced child and adolescent psychiatrists or clinical child psychologists, based on the information from direct interviews with the patient or care givers, information by the multi-professional team and behaviour observation. Comorbid diagnoses according to ICD-10 [8] were depressive episodes or recurrent depressive disorder $(n=24)$, other anxiety disorders $(n=7)$, eating disorders $(n=2)$, somatoform disor$\operatorname{der}(n=2)$, developmental disorder of speech $(n=3)$, reading disorder $(n=1)$ and attention-deficit hyperactivity disorder $(n=1)$. Six of the patients received antidepressant medication (Sertralin, Citalopram, Escitalopram) while taking part in our study. The CG included 24 typically developing youth ( $M$ age $=15.68$ years, $\mathrm{SD}=2.04$ years, $54 \%$ female).

\section{Procedure}

The study was approved by the local ethical committee of the Medical Faculty of the Goethe University Frankfurt am Main. Written informed consent was obtained from both parents and youth. First, participants received paper-pencil questionnaires once consent had been obtained and returned the completed questionnaires. Subsequently, the participants took part in an experimental session and completed the IAT [37] and the AMP [38]. To conduct a manipulation check of the stimuli used in the AMP [38], 35 of the participants (12 patients, 17 controls) completed an explicit rating of the face stimuli using the Self-Assessment Manikin Rating (SAM) afterwards [52, 53].

\section{Measures-Experimental tasks}

The Implicit Association Test (IAT [32]) is a computerbased reaction time paradigm measuring implicit associations between target and attribute categories. In this study, we used the IAT for implicit social assumptions (IAT-SP), similar to the study by Wong and colleagues [46] in adults. The IAT-SP investigates the target categories of self (I, own, my, me, self) and other (them, others, you, your, they) and the attribute categories of rejection (forgotten, alienated, deserted, shunned, disliked) and acceptance (loved, welcomed, admired, included, respected). The participants were instructed to assign a word belonging to either a target category or an attribute category (e.g. "me" as representing target category "self") presented in the middle of the screen to the correct one of the opposing categories presented in the left and right upper corners of the screen (e.g. left: "self" vs. right: "other") as fast as possible. The participants used keys on the keyboard to assign the words to the categories with left-sided keys corresponding to the category in the left corner and right-sided keys to the right corner (e.g. "me" $=>$ "self" in left corner $=>$ left button press). When the answer was incorrect a red " $X$ " appeared and they were asked to assign it to the correct category. Within the IATSP there were five blocks of trials. The first, second and fourth blocks were practise blocks, where one category was presented in each upper corner (e.g. left: "self" vs. right: "other"). Each practise block consisted of 24 trials. The third and fifth blocks were critical test blocks, where category combinations (e.g. "self + acceptance" in upper left corner; "other + rejection" in upper right corner) were presented and the word displayed in the middle of the screen had to be assigned to one of the category combinations (e.g. "me" $=>$ "self + acceptance"). The sides of the presented category combinations were switched between the third and fifth blocks. Each test block consisted of 24 practise trials and 40 test trials. Four different versions of the IATSP existed to counterbalance all possible combinations of the categories (e.g. "self", "other" and "acceptance", "rejection") and sides (right or left upper corner). The versions are presented in the supplement (Online Resource 1). The internal consistencies of the reaction times of the IAT-SP in our study ranged from Cronbach's $\alpha=0.83$ to $\alpha=0.94$.

For the IAT, the $\mathrm{D}_{1}$ score as an IAT effect was computed according to the published algorithm [54]. All response latencies $>10.000 \mathrm{~ms}$ and $<300 \mathrm{~ms}$ were first deleted and response latencies for errors were included in the calculation. All response latencies of practise and test trials of the critical blocks (blocks 3 and 5) were used. The mean latencies for the critical blocks for each IAT and the individualrespondent standard deviations were computed first. In the next step, the mean response latencies for the "Self/Rejection" associations were subtracted from the "Other/Rejection" mean response latencies. This difference was then divided by the pooled standard deviation to obtain the $\mathrm{D}_{1}$ score. Higher values in the IAT $\mathrm{D}_{1}$ score, thus, correspond to a higher implicit assumption of feeling socially rejected.

The Affect Misattribution Procedure (AMP [33]) is an implicit computer-based paradigm that combines the procedure of computer-based priming experiments and the logic of projective tests. The AMP taken in this study was adapted for social stimuli [38]. In the AMP [38], primes (pictures of faces) are presented supraliminally to the participants and, subsequently, an ambiguous target stimulus (Chinese character) is shown; the prime was presented for $33 \mathrm{~ms}$, followed by a blank screen for $466 \mathrm{~ms}$, the ambiguous stimulus for $117 \mathrm{~ms}$ and a mask sign (black and white pattern) during 
which the participants had to answer. All stimuli were presented in the centre of the screen. The participants were told to rate the ambiguous stimulus on a 4-point Likert scale ranging from very unpleasant (1) to very pleasant (4) and were directed to ignore the prime. The primes used were pictures of young adults showing angry, fearful, happy or neutral emotional facial expressions (taken from the Radboud Faces Database) [55]. The AMP [38] consisted of 38 trials for each of the four emotional facial expressions [4 emotions (angry, fearful, happy, neutral) $\times 38=152$ trials], half male and half female pictures. Across six previous studies, the AMP showed a mean Cronbach's $\alpha=0.88$ [38]. In our study, the internal consistencies for the AMP ranged from $\alpha=0.77$ to $\alpha=0.95$. For the AMP, we assessed the pleasantness of the Chinese characters for each emotional face category (angry, fear, happy, neutral) by computing the pleasantness mean score for each emotion separately and additionally computed a pleasantness total score by calculating the mean of the pleasantness responses for all four emotions for each participant.

In addition, explicit ratings (valence and arousal) of the emotional faces were assessed to examine the validity of the prime stimuli used in the AMP. A subsample $(N$ patients $=14, N$ controls $=21$ ) rated the valence and arousal of all the primes. The emotional facial expressions were presented to the participants in the middle of the screen and the participants were asked to rate the prime first on valence (nine pictures ranging from sad to happy, with a scale of 1-9 underneath) and, second, on arousal (nine pictures ranging from calm to excited, with a scale of 1-9 underneath) using the non-verbal Self-Assessment Manikin (SAM [52]).

\section{Measures-Questionnaires}

The Diagnostic System for Mental Disorders (DISYPS-II [56]) is a German rating system to assess the diagnosis and severity of different mental disorders in childhood and youth according to ICD-10 and DSM-5. For our study, the total score of the 33-item self-report questionnaire for anxiety disorders ( $S B B-A N Z)$ and the social anxiety subscale score were used to measure the severity of explicit general anxiety and social anxiety. All items were answered on a fourpoint Likert scale from $0=$ "not at all" to $3=$ "particularly true". The total scale showed good convergent and discriminant validity and acceptable to high internal consistency $(\alpha=0.59-0.92)$ [56, 57]. In our study, internal consistencies for the SBB-ANZ total score were Cronbach's $\alpha=0.85$ for the SPG and Cronbach's $\alpha=0.79$ for the CG.

The German version of the Social Phobia and Anxiety Inventory for Children (SPAI-C [58]) is a 26-item selfreport assessing the severity of social anxiety in children and youth. Somatic, cognitive and behavioural aspects of social anxiety are rated on a 3-point Likert-scale ranging from "never" to "mostly/always". Very good internal consistency ( $\alpha=0.92-0.95)$ and convergent and discriminant validity could be shown for clinical and non-clinical samples [59, 60]. In our study, internal consistencies of $\alpha=0.95$ for the SPG and $\alpha=0.92$ for the controls were obtained.

The Beck's Depression Inventory (BDI-II [61]) is a self-report measure assessing the severity of depressive symptoms. The 21-item questionnaire is based on DSMIV criteria and showed very good internal consistencies $(\alpha=0.84-0.94)$ in clinical and non-clinical samples and sufficient retest-reliability ( $\alpha>0.75$ ) [62]. In addition, the BDI-II shows high convergent and content validity $[62,63]$. In our study, the internal consistencies of the BDI-II for patients were $\alpha=0.93$ and for controls $\alpha=0.72$.

The following questionnaires were obtained from the typically developing controls to check the exclusion criteria.

The German version of the Youth Self Report (YSR 11-18 [50]) is a self-report questionnaire for youth aged 11-18 years. The items of the YSR 11-18 can be divided into eight problem scales (anxious/depressed, withdrawn/ depressed, somatic complaints, social problems, thought problems, attention problems, rule-breaking behaviour and aggressive behaviour). In the present study, internal consistencies for the subscales ranged from $\alpha=0.86$ to $\alpha=0.91$.

The German version of the Child Behavior Checklist (CBCL 6-18 [51]) is a questionnaire for parents of children and youth aged 6-18 years with the same subscales as the YSR 11-18, assessing symptoms of the children from the parents' point of view. In our study, the internal consistencies of the subscales ranged from $\alpha=0.62$ to $\alpha=0.99$.

\section{Statistics}

All dependent variables were normally or approximately normally distributed. Descriptive data were compared by analysis of variance (ANOVA, i.e., age, IQ, BDI-II, SPAI$\mathrm{C}$ and SBB-ANZ) or the Chi-square test (i.e., sex). To test the study's hypotheses, analyses of covariance (ANCOVA) with the $\mathrm{D}_{1}$ scores of the IAT-SP, the pleasantness total score of the AMP, or the explicit valence or explicit arousal, as dependent measures, were computed. Furthermore, we computed repeated measures ANCOVAs (rmANCOVA) with the AMP's pleasantness score for each emotion separately, explicit valence ratings for each emotion separately and the explicit arousal ratings for each emotion separately, as dependent measures. The factor group was the predictor of interest, while the covariates were BDI-II and IQ in the rmANCOVAs. Due to some missing values of the covariates intelligence and depressive symptoms, the number of participants in the ANCOVA was slightly reduced compared to recruitment. For the AMP, initially, a model without the task related factor "emotions" was calculated, followed by a sensitivity analysis to test for group $\times$ emotions interaction. 
Bonferroni-adjusted post-hoc analyses were computed. Greenhouse-Geisser correction was used to correct for violations of sphericity and partial eta-square was calculated as effect size $\left(\eta_{p}^{2} \geq 0.01\right.$ small effect; $\eta_{p}^{2} \geq 0.06$ medium effect; $\eta_{p}^{2} \geq 0.14$ large effect). $P$ values $<0.05$ were taken as criteria for rejection of $\mathrm{H} 0$. Due to the sample size which had a power of $80 \%$ to detect only a large effect size with an alpha $<0.05$, no adjustment for multiple testing was done. To explore the role of depressive symptoms, the ANCOVAs of IAT-SP and AMP were repeated without BDI-II as the covariate. In addition, correlations between the implicit and explicit measures were assessed by computing partial correlations, controlling for the covariates BDI-II and IQ. Relevant correlations above $|r \geq 0.3|$ are discussed below.

\section{Results}

\section{Participant characteristics}

Demographic characteristics and questionnaire data of the SPG and the CG are presented in Table 1. The SPAI-C total score $\left[F(1,43)=82.25, p<0.001, \eta_{p}^{2}=0.66\right]$ and SBB-ANZ total score $\left[F(1,38)=69.20, p<0.001, \eta_{p}^{2}=0.65\right]$ were higher in the SPG than the CG. The groups did not differ with regard to sex $\left[\chi^{2}(1)=2.21 ; p=0.138, \Phi=0.21\right]$ or age $\left[F(1,49)=0.05, p=0.832, \eta_{p}^{2}=0.01\right]$. However, there were significant differences regarding IQ $[F(1,46)=4.21$, $\left.p=0.046, \eta_{p}^{2}=0.08\right]$ and depressive symptoms (BDI-II) $\left[F(1,48)=67.43, p<0.001, \eta_{p}^{2}=0.58\right]$. Therefore, IQ and BDI were used as covariates in further analyses.

\section{Experimental measures (IAT and AMP)}

Results of the IAT and AMP by group are shown in Table 2. The ANCOVA for the IAT-SP showed that both groups

Table 1 Sample description

\begin{tabular}{lllll}
\hline Characteristics & SPG $(N=27)$ & CG $(N=24)$ & \multicolumn{2}{l}{ Statistics } \\
\cline { 4 - 5 } & $M(\mathrm{SD})$ & $M(\mathrm{SD})$ & $p$ & Effect size \\
\hline Age (years) & $15.58(1.29)$ & $15.68(2.04)$ & .832 & $\eta_{p}^{2}=.01$ \\
Sex (\% female) & $74.1 \%$ & $54.2 \%$ & .138 & $\Phi=.21$ \\
IQ & $102(11.9)$ & $109(10.9)$ & .046 & $\eta_{p}^{2}=.08$ \\
BDI-II (0-63) & $27.22(13.85)$ & $3.46(2.87)$ & $<.001$ & $\eta^{2}=.58$ \\
SPAI-C (0-52) & $31.34(12.40)$ & $4.23(4.98)$ & $<.001$ & $\eta_{p}^{2}=.66$ \\
SBB-ANZ (0-99) & $10.75(5.76)$ & $0.52(0.98)$ & $<.001$ & $\eta_{p}^{2}=.65$ \\
\hline
\end{tabular}

$S P G$ Social Phobia group; $C G$ Control group; $M$ Mean score; $S D$ Standard deviation; $N$ Number of participants; BDI-II Beck's Depression Inventory; SPAI-C Social Phobia and Anxiety Inventory for children/adolescents; $S B B-A N Z$ total score of the self-report inventory for Anxiety Disorders; effect sizes $\left(\eta_{p}^{2} \geq .01\right.$ small effect; $\eta_{p}^{2} \geq .06$ medium effect; $\eta_{p}^{2} \geq .14$ large effect)
Table 2 Cognitive test results and self-assessment by group-raw data

\begin{tabular}{lcc}
\hline & SPG $(N=27)$ & CG $(N=24)$ \\
& $M(\mathrm{SD})$ & $M(\mathrm{SD})$ \\
\hline IAT-SP D 1 score & $-.12(.45)$ & $-.29(.23)$ \\
AMP pleasantness total score & $2.31(.32)$ & $2.64(.43)$ \\
AMP angry & $2.11(.47)$ & $2.55(.46)$ \\
AMP fear & $2.17(.44)$ & $2.61(.47)$ \\
AMP happy & $2.67(.54)$ & $2.76(.43)$ \\
AMP neutral & $2.30(.39)$ & $2.65(.39)$ \\
SAM valence total score & $4.76(.51)$ & $4.88(.39)$ \\
SAM valence angry & $3.70(.95)$ & $4.00(.84)$ \\
SAM valence fear & $3.76(.77)$ & $4.09(.72)$ \\
SAM valence happy & $7.22(.99)$ & $6.77(.90)$ \\
SAM valence neutral & $4.37(.75)$ & $4.65(.45)$ \\
SAM arousal total score & $5.41(.83)$ & $5.21(1.47)$ \\
SAM arousal angry & $6.37(1.00)$ & $4.58(1.75)$ \\
SAM arousal fear & $6.58(1.13)$ & $5.14(1.94)$ \\
SAM arousal happy & $4.43(1.96)$ & $3.84(1.63)$ \\
SAM arousal neutral & $4.27(1.46)$ & $3.27(1.26)$ \\
\hline
\end{tabular}

$S P G$ Social Phobia group; $C G$ Control group; $M$ Mean score; $S D$ Standard deviation; $N$ number of participants; IAT-SP $D_{l}$ score $\mathrm{D}_{1}$ score of the Implicit Association Test; AMP angry, fear, happy, neutral Pleasantness scores in the Affective Misattribution Procedure for each emotion (angry, fear, happy, neutral) separately, SAM valence angry, fear, happy, neutral Valence scores in the explicit rating using the Self-Assessment Manikin for each emotion separately (angry, fear, happy, neutral); SAM arousal angry, fear, happy, neutral Arousal scores in the explicit rating using the Self-Assessment Manikin for each emotion separately (angry, fear, happy, neutral)

did not differ regarding their implicit assumption of being rejected $\left[F(1,41)=0.92, p=0.343, \eta^{2}{ }_{p}=0.022\right]$. When the ANCOVA was repeated without BDI-II as the covariate, a difference with a medium effect size between SPG and CG was found $\left[F(1,43)=2.94, p=0.094, \eta_{p}^{2}=0.064\right]$. This is reflected by the raw mean scores between groups (SPG $\mathrm{D}_{1}$-score: $M=-0.12, S D=0.45$; $\mathrm{CG} \mathrm{D}_{1}$-score: $M=-0.29$, $S D=0.23$ ). This suggests greater assumptions of being rejected due to depressive symptoms, but not due to anxiety disorder, in patients with SP and depressive symptoms compared to healthy controls. Regarding the AMP, the ANCOVA showed differences between the pleasantness total scores of the groups $\left[F(1,39)=6.19, p=0.017, \eta_{p}^{2}=0.137\right]$. The SPG showed lower pleasantness total scores $(M=2.31$, $\mathrm{SD}=0.32)$ than the $\mathrm{CG}(M=2.64, \mathrm{SD}=0.43)$, which could be interpreted as a higher implicit interpretation bias in the SPG than in the CG. Repeating the ANCOVA without the factor BDI-II as the covariate did not change the results $[F(1$, $\left.40)=5.55, p=0.024, \eta_{p}^{2}=0.122\right]$. Adding the factor "emotion" to the rmANCOVA resulted in a significant main effect of emotion $\left[F(1.6,60.4)=3.50, p=0.048, \eta^{2}{ }_{p}=0.082\right]$. Pleasantness scores for the emotions were shown to be 
rated differently across all patients. However, no interaction of emotion and group $[F(1.6,60.4)=0.39, p=0.628$, $\left.\eta_{p}^{2}=0.010\right]$ occurred and, again, group differences did not change $\left[F(1,39)=6.19, p=0.017, \eta_{p}^{2}=0.137\right]$ underlining the finding that a difference in the pleasantness score between the SPG and CG was independent of emotions.

\section{Explicit valence and arousal ratings (SAM) for facial expressions in the AMP}

Results of the explicit valence and arousal ratings by group are shown in Table 2. Regarding the valence ratings, the ANCOVA could not demonstrate differences between the groups $\left[F(1,25)=0.47, p=0.498, \eta_{p}^{2}=0.019\right]$. The rmANCOVAs showed no main effect of valence $[F(1.7$, $\left.42.3)=1.60, p=0.216, \eta^{2}{ }_{p}=0.06\right]$, no main effect of group $\left[F(1,25)=0.47, p=0.498, \eta_{p}^{2}=0.019\right]$ and no interaction effect of group and emotion $[F(1.7,42.3)=0.17, p=0.807$, $\left.\eta_{p}^{2}=0.007\right]$. The emotional faces were not rated differently on valence when participants were asked to rate them explicitly and the groups did not differ with regard to their valence rating. The results did not change when repeating the analyses without BDI-II as a covariate. With regard to arousal, the ANCOVA revealed no differences between the groups $\left[F(1,25)=0.56, p=0.463, \eta_{p}^{2}=0.022\right]$. The rmANCOVA showed no main effect of arousal $[F(2.1,51.4)=0.24$, $\left.p=0.798, \eta_{p}^{2}=0.009\right]$ and no interaction effect was found $\left[F(2.1,51.4)=0.53, p=0.599, \eta_{p}^{2}=0.021\right]$. In addition, when explicitly rating the faces on arousal, no differences for the various emotional faces were obtained and no differences between the CG and SPG occurred. When repeating the analyses without BDI-II as a covariate, a group difference between the SPG and CG was found $[F(1,26)=8.02$, $\left.p=0.009, \eta^{2}{ }_{p}=0.236\right]$ suggesting a difference in the rating of arousal between youth with SP and depressive symptoms and healthy controls.

\section{Correlations of implicit and explicit social phobia measures}

With regard to correlations of IAT-SP and explicit social phobia measures across all participants (SPG and CG), correlations above $|r=0.30|$ were observed between IATSP and SPAI-C $(r=0.530, p=0.002 ; \mathrm{CI}=[0.31 ; 0.87])$, IAT-SP and the social phobia subscale of the SBB-ANZ $(r=0.53, p=0.002 ; \mathrm{CI}=[0.31 ; 0.87])$ and IAT-SP and the SBB-ANZ total score $(r=0.364, p=0.041, \mathrm{CI}=[0.10$; $0.66])$. For the SPG, positive correlations above $|r=0.30|$ were shown between IAT-SP and SPAI-C $(r=0.54$, $p=0.039 ; \mathrm{CI}=[0.32 ; 0.88])$, IAT-SP and the social phobia subscale of the SBB-ANZ $(r=0.51, p=0.055 ; \mathrm{CI}=[0.28$; $0.84])$ and IAT-SP and the SBB-ANZ total score $(r=0.35$, $p=0.20, \mathrm{CI}=[0.09 ; 0.65])$. For the CG separately, correlations above $\mathrm{I}=0.3 \mathrm{l}$ were found between IAT-SP and SPAI-C ( $r=0.46, p=0.082 ; \mathrm{CI}=[0.22 ; 0.78])$, IAT-SP and the social phobia subscale of the SBB-ANZ $(r=0.44$, $p=0.101 ; \mathrm{CI}=[0.19 ; 0.75])$ and IAT-SP and the SBBANZ total score $(r=0.45, p=0.097, \mathrm{CI}=[0.20 ; 0.76])$. For AMP pleasantness, the total score and explicit social phobia measures across all participants revealed no correlations above $|r=0.3|$, whereas for the SPG separately, correlations above $|r=0.3|$ were observed between the AMP pleasantness total score and SPAI-C total score $(r=-0.50$, $p=0.057, \mathrm{CI}=[-0.83 ;-0.27])$ and between AMP pleasantness total score and the SBB-ANZ total score $(r=-0.39$, $p=0.146, \mathrm{CI}=[-0.69 ;-0.13])$. For the CG separately, correlations above $|r=0.3|$ were shown for the AMP pleasantness total score and SBB-ANZ $(r=0.43, p=0.113$, $\mathrm{CI}=[0.18 ; 0.74])$. All correlations are shown in Table 3.

Table 3 Partial correlations between implicit and explicit measures (self-reports)

\begin{tabular}{|c|c|c|c|c|c|c|c|c|c|c|c|c|c|c|c|}
\hline & \multicolumn{3}{|c|}{ IAT-SP } & \multicolumn{3}{|l|}{ AMP } & \multicolumn{3}{|c|}{ SPAI-C } & \multicolumn{3}{|c|}{$\begin{array}{l}\text { SBB-ANZ (SP sub- } \\
\text { scale) }\end{array}$} & \multicolumn{3}{|c|}{ SBB-ANZ } \\
\hline & SPG & CG & Total & SPG & CG & Total & SPG & CG & Total & SPG & CG & Total & SPG & CG & Total \\
\hline 1. IAT-SP & 1 & 1 & 1 & & & & & & & & & & & & \\
\hline 2. AMP & -.17 & -.17 & -.27 & 1 & 1 & 1 & & & & & & & & & \\
\hline 3. SPAI-C & $.54 *$ & .46 & $.53^{* *}$ & -.50 & .10 & -.27 & 1 & 1 & 1 & & & & & & \\
\hline 4. SBB-ANZ (SP subscale) & .51 & .44 & $.53 * *$ & -.28 & -.04 & -.22 & $.76 * *$ & .41 & $.76 * * *$ & 1 & 1 & 1 & & & \\
\hline 5. SBB-ANZ (total score) & .35 & .45 & $.36^{*}$ & -.39 & .43 & -.09 & $.70 * *$ & .31 & $.69^{* * * *}$ & $.71^{* *}$ & $.52 *$ & $.73 * * *$ & 1 & 1 & 1 \\
\hline
\end{tabular}

SP Social Phobia; SPG Social Phobia group; $C G$ Control group; total all participants; IAT-SP Implicit Association Test (social phobia version); $A M P$ total score Affective Misattribution Procedure total pleasantness score; SPAI-C Social Phobia and Anxiety Inventory for children/adolescents; $S B B$-ANZ SP subscale score of the self-report inventory for Anxiety Disorders' social phobia subscale; $S B B$ - $A N Z$ total score of the selfreport inventory for Anxiety Disorders

$* p<.05, * * p<.01, * * * p<.001$ 


\section{Discussion}

The aim of the study was to investigate implicit, disorderspecific, dysfunctional assumptions and interpretation bias in youth with severe, chronic SP. So far, these cognitive processes have not been investigated in such a severely affected group of youth and this study may contribute to closing this research gap. The scores in the self-reports confirmed the severity of the SP. Compared to other studies assessing youth with SP, our sample showed higher SPAI-C total scores [64, 65]. Furthermore, depressive symptoms were higher than in other comparable studies on youth with SP [45]. The controls showed similar mean SPAI-C and BDI-II scores compared to other studies with non-clinical samples [45, 64, 65].

We hypothesised that youth with severe, chronic SP showed stronger implicit assumptions about oneself of being rejected than healthy controls. The results of our study indicate no differences between the groups for the implicitly assessed assumptions of being rejected as measured by the IAT-SP. This result is in line with another study [46] which also found no differences between socially anxious adults and controls regarding implicit assumptions about being rejected. However, adults with SP and comorbid depression showed stronger implicit dysfunctional social assumptions compared to SP without comorbid depression and the controls [46]. When not controlling for depressive symptoms in our study, results similarly showed group differences with a medium effect size. It is worth mentioning that we examined a rather small sample, thus, the observed medium effect group difference observed when not controlling for depressive symptoms may have reached significance in a larger sample. Therefore, feelings of being rejected may be due to the frequently co-occurring depression in youth and adult SP. Rejection sensitivity has been discussed as a specific aspect for SP [47] and for depressive disorder [66, 67]. Given studies in depressed youth which replicated rejection sensitivity as a risk factor for depression [68-70], our results have to be interpreted as follows. We cannot confirm the hypothesis that the feeling of being rejected is specific to SP, but it likely plays a strong role in co-occurring depression in SP. The observed positive correlation of the explicit self-reported severity of SP (SPAI-C and SBBANZ social phobia subscale) with the implicit assumptions of being rejected (IAT-SP) were driven by the SPG. The highest correlations of the implicit assumptions of being rejected and explicit SP symptoms and anxiety, in general, were found in the SPG. The higher the level of SP symptoms and anxiety, the stronger the implicit assumption of social rejection seems to exist, but this connection is more likely to be present above a certain level of social phobia.
Second, we hypothesised that youth with SP compared to healthy controls show a stronger implicit interpretation bias, resulting in the more negative interpretation of emotional faces. In accordance with our hypothesis, the SP group showed lower pleasantness responses than the control group over all primes with a large effect which was independent of comorbid depressive symptoms. These results are in accordance with findings of a meta-analysis in typically developing children and youth predominantly from community-based samples which found a medium to large, albeit heterogeneous, effect for positive associations of anxiety with negative interpretation bias assessed with self-report measures [71]. In the meta-analysis, age and content specificity moderated the effect sizes which were found to be larger in older individuals and when the scenario content matched the anxiety subtype. Similar to our study, comorbid psychiatric disorders or symptoms, such as depressive symptoms, did not change this finding. The present study supplements the meta-analysis by replicating the anxiety-related interpretation bias also for an implicit disorder specific task, as we tested for the pleasantness of facial expressions. The hypothesised negative correlations for the AMP total score and the explicit SPAI-C rating were only shown in the SPG. Lower pleasantness ratings of facial expressions were associated with higher explicit SP symptoms in SPAI-C. In the CG this association could not be observed; this supports the specificity of this correlation for SP and the validity of the cognitive theory of SP. The negative facial expressions may have triggered negative dysfunctional assumptions in SP and, thus, may be relevant as a risk factor for SP and for the maintenance of SP. The exact mechanisms need to be studied by longitudinal studies with a different design, as, from this study, we cannot differentiate if interpretation bias is a symptom of manifest SP or a risk factor. Furthermore, for SPG, a negative correlation for AMP total scores and the rating of general anxiety (SBB-ANZ) was observed. Lower pleasantness total scores in the AMP were associated with higher explicitly reported general anxiety. Since AMP total scores correlated with explicit SP symptoms and general anxiety, it is important to keep in mind that we cannot differentiate whether the group differences in the AMP were driven by social anxiety or by general anxiety. Future research with a larger sample size is needed to differentiate the differences more precisely. Studies on modifying interpretation bias using Cognitive Bias Modification (CBM) interventions, e.g. the Interpretation Modification Program (IMP) or CBM using positive imagery, were found to be successful in altering interpretation bias in SP and depression [72, 73]. In these studies, interpretation bias was assessed explicitly using self-report measures. To our knowledge, so far, no studies exist on the modification of interpretation bias by assessing it implicitly. 
Methodically, the explicit ratings of the facial expressions did not result in the expected differences in valence and arousal between the different facial expressions, although in a validation study on these face stimuli, the Radboud Faces Data Set [55], participants recognised the displayed emotions correctly and rated their valence according to the expectation of the authors on a positive to negative continuum. In our study, participants were asked to rate valence on a SAM rating scale [52] from happy to sad. It is possible that these categories were not suitable to assess differences in valence, whereas other categories, e.g. positive to negative (as used in the validation study [55]), would have been more suitable to find differences. Second, the missing differences could also be due to the small subsample size. Thirdly, the unexpected results of arousal and valence ratings of the facial expressions could be due to less developed emotion recognition skills of youth with SP. It was found that children were less accurate in recognising the expressed emotion of the Radboud Faces [55] than adults [74]. Recognition of emotional facial expression is considered to develop with age and is also proposed to depend on the emotion expressed [75]. Since patients with SP show a tendency to avoid emotional faces when social threat is activated [76] and the onset of SP in our sample is considered early, it could be argued that this has resulted in an impairment of the ability to recognise emotional facial expression, thus, this may have led to the missing differences in arousal and valence ratings.

Several limitations should be mentioned, in particular, the rather small sample size which only allowed the detection of large group differences with a power of $80 \%$ and an alpha $<0.05$. Future research will be needed with larger sample sizes to support our results. Similar to other studies on social phobia in youth, many individuals with severe, chronic SP, who were asked to participate in the study, declined participation due to their general anxiety or anxiety related to study procedures (e.g. despite detailed information, an uncertainty about what exactly they could expect possibly existed). Therefore, selection biases for participation cannot be ruled out, since factors, such as parental control or personality aspects, were not controlled for. Another limitation linked to the small sample size is that it was not possible to control for medication. Six of the participants had antidepressant medication when participating; exclusion of these participants would have led to an even smaller sample size. Antidepressant medication is unlikely to account for the effects found in this study, but may potentially have decreased the effect sizes. Another limitation concerning the sample is the possibility of other comorbid diagnoses, besides depression, influencing the results. It was not possible to control for all comorbidities due to the small sample size. By controlling for depression, we have ruled out one major influencing factor. Other comorbidities were found in in a very small number of SP participants, such as eating disorder ( $n=2$; weight $>10$. BMI-percentile), somatoform disorder $(n=2)$, and $\operatorname{ADHD}(n=1)$. As these numbers are small, and individuals with a history of an eating disorder showed a BMI-percentile $>10$, it seems to be unlikely that group differences may be driven by these comorbid disorders. Given the relatively high comorbidity with other anxiety disorders [77], we cannot completely rule out that group differences may be driven by anxiety in general, and not solely by social phobia.

In addition, due to the cross-sectional design of our study, we cannot draw any causal conclusions. Therefore, for future research, longitudinal studies can be used to test whether, for example, dysfunctional assumptions are relevant as risk factors for and precede the development of clinical SP. For the implicit interpretation it would be interesting to see whether the implicit interpretation bias could also be altered by interpretation bias modification interventions or other cognitive interventions.

Taken together, implicit interpretation bias is more likely to play a role in SP, whereas implicit dysfunctional social assumptions of being rejected were not confirmed as being specific to SP, but rather for co-occurring depressive symptoms in SP. A high number of explicitly assessed symptoms of social phobia and anxiety, in general, were associated with higher implicit assumptions of being rejected and lower pleasantness ratings of facial expressions in youth with SP, when controlled for depressive symptoms. However, these results need to be interpreted with regard to the possible influence of general anxiety, since this could not be assessed separately in the present study. Other limitations of the study, e.g. the small sample size, should also be considered. Since it remains unclear whether implicit assumptions and biases are the cause, consequence or epiphenomena of SP, future research should take a closer look at causal conclusions and modification possibilities of the implicit cognitive aspects in SP.

Supplementary Information The online version contains supplementary material available at https://doi.org/10.1007/s00787-021-01879-3.

Funding Open Access funding enabled and organized by Projekt DEAL.

\section{Declarations}

Conflict of interest CMF receives royalties for books on ASD, ADHD and MDD. She receives research funding for studies on ASD and ADHD by the EU, the BMBF and the DFG. The other authors declare that they have no conflict of interest.

Ethical approval The study was approved by the local ethical committee of the Medical Faculty of the Goethe University Frankfurt am Main and has, therefore, been performed in accordance with ethical standards laid down in the 1964 Declaration of Helsinki and its later 
amendments. Written informed consent was obtained from participants and their parents.

Open Access This article is licensed under a Creative Commons Attribution 4.0 International License, which permits use, sharing, adaptation, distribution and reproduction in any medium or format, as long as you give appropriate credit to the original author(s) and the source, provide a link to the Creative Commons licence, and indicate if changes were made. The images or other third party material in this article are included in the article's Creative Commons licence, unless indicated otherwise in a credit line to the material. If material is not included in the article's Creative Commons licence and your intended use is not permitted by statutory regulation or exceeds the permitted use, you will need to obtain permission directly from the copyright holder. To view a copy of this licence, visit http://creativecommons.org/licenses/by/4.0/.

\section{References}

1. Polanczyk GV, Salum GA, Sugaya LS et al (2015) Annual research review: a meta-analysis of the worldwide prevalence of mental disorders in children and adolescents. J Child Psychol Psychiatry 56(3):345-365. https://doi.org/10.1111/jcpp.12381

2. Burstein M, He J-P, Kattan G et al (2011) Social phobia and subtypes in the national comorbidity survey-adolescent supplement: prevalence, correlates, and comorbidity. J Am Acad Child Adolesc Psychiatry 50(9):870-880. https://doi.org/10.1016/j.jaac.2011.06. 005

3. Merikangas KR, He J-P, Burstein M et al (2010) Lifetime prevalence of mental disorders in U.S. adolescents: results from the National Comorbidity Survey Replication-Adolescent Supplement (NCS-A). J Am Acad Child Adolesc Psychiatry 49(10):980 989. https://doi.org/10.1016/j.jaac.2010.05.017

4. Wittchen H-U, Fehm L (2003) Epidemiology and natural course of social fears and social phobia. Acta Psychiatr Scand 108:4-18

5. Ihle W, Esser G (2002) Epidemiologie psychischer Störungen im Kindes- und Jugendalter: Prävalenz, Verlauf, Komorbidität und Geschlechtsunterschiede [Epidemiology of mental disorders in childhood and adolescence: Prevalence, course, comorbidity and gender differences]. Psychologische Rundschau 53(4):159-169. https://doi.org/10.1026/0033-3042.53.4.159

6. Organization WH (2004) International statistical classification of diseases and related health problems. World Health Organization

7. van Ameringen M, Mancini C, Farvolden P (2003) The impact of anxiety disorders on educational achievement. J Anxiety Disord 17(5):561-571

8. Waghorn G, Chant D, White P et al (2005) Disability, employment and work performance among people with ICD-10 anxiety disorders. Aust N Z J Psychiatry 39(1-2):55-66

9. Copeland WE, Angold A, Shanahan L et al (2014) Longitudinal patterns of anxiety from childhood to adulthood: the Great Smoky Mountains Study. J Am Acad Child Adolesc Psychiatry 53(1):21-33

10. Leigh E, Clark DM (2018) Understanding social anxiety disorder in adolescents and improving treatment outcomes: applying the cognitive model of Clark and Wells (1995). Clin Child Fam Psychol Rev 21(3):388-414. https://doi.org/10.1007/ s10567-018-0258-5

11. Clark DM, Wells A (1995) A cognitive model of Social Phobia. In: Heimberg RG (ed) Social Phobia: Diagnosis, Assessment, and Treament. Guilford Press, pp 69-74

12. Melfsen S, Florin I (2002) Do socially anxious children show deficits in classifying facial expressions of emotions? J Nonverbal Behav 26(2):109-126
13. Tanner RJ, Stopa L, de Houwer J (2006) Implicit views of the self in social anxiety. Behav Res Ther 44(10):1397-1409

14. Schreiber F, Höfling V, Stangier U et al (2012) A cognitive model of social phobia: applicability in a large adolescent sample. Int J Cogn Ther 5(3):341-358

15. Clark DM (2005) A Cognitive Perspective on Social Phobia. In: Crozier WR, Alden LE (eds) The essential handbook of social anxiety for clinicians. John Wiley \& Sons Ltd., pp 193-218

16. Castillo MD, Leandro PG (2010) Interpretation bias in anxiety a synthesis of studies with children and adolescents. Procedia Soc Behav Sci 5:1105-1111. https://doi.org/10.1016/j.sbspro.2010.07. 243

17. Stopa L, Clark DM (2001) Social phobia: comments on the viability and validity of an analogue research strategy and British norms for the fear of negative evaluation questionnaire. Behav Cogn Psychother 29(4):423-430

18. Vassilopoulos SP, Banerjee R (2008) Interpretations and judgments regarding positive and negative social scenarios in childhood social anxiety. Behav Res Ther 46(7):870-876

19. Voncken MJ, Bögels SM, de Vries K (2003) Interpretation and judgmental biases in social phobia. Behav Res Ther 41(12):1481-1488

20. Stopa L, Clark DM (2000) Social phobia and interpretation of social events. Behav Res Ther 38(3):273-283

21. Amir N, Foa EB, Coles ME (1998) Automatic activation and strategic avoidance of threat-relevant information in social phobia. J Abnorm Psychol 107(2):285

22. Miers AC, Blöte AW, Bögels SM et al (2008) Interpretation bias and social anxiety in adolescents. J Anxiety Disord 22(8):14621471. https://doi.org/10.1016/j.janxdis.2008.02.010

23. Haller SPW, Raeder SM, Scerif G et al (2016) Measuring online interpretations and attributions of social situations: Links with adolescent social anxiety. J Behav Ther Exp Psychiatry $50: 250-256$

24. Tsuji Y, Shimada S (2018) Socially anxious tendencies affect impressions of others' positive and negative emotional gazes. Front Psychol 9:2111. https://doi.org/10.3389/fpsyg.2018.02111

25. Laposa JM, Cassin SE, Rector NA (2010) Interpretation of positive social events in social phobia: an examination of $\operatorname{cog}$ nitive correlates and diagnostic distinction. J Anxiety Disord 24(2):203-210

26. Joormann J, Gotlib IH (2006) Is this happiness I see? Biases in the identification of emotional facial expressions in depression and social phobia. J Abnorm Psychol 115(4):705

27. Hunter LR, Buckner JD, Schmidt NB (2009) Interpreting facial expressions: the influence of social anxiety, emotional valence, and race. J Anxiety Disord 23(4):482-488

28. Heuer K, Lange W-G, Isaac L et al (2010) Morphed emotional faces: emotion detection and misinterpretation in social anxiety. J Behav Ther Exp Psychiatry 41(4):418-425

29. Morrison AS, Heimberg RG (2013) Social anxiety and social anxiety disorder. Annu Rev Clin Psychol 9:249-274

30. Beevers CG (2005) Cognitive vulnerability to depression: a dual process model. Clin Psychol Rev 25(7):975-1002. https://doi. org/10.1016/j.cpr.2005.03.003

31. Wiers RW, Bartholow BD, van den Wildenberg E et al (2007) Automatic and controlled processes and the development of addictive behaviors in adolescents: a review and a model. Pharmacol Biochem Behav 86(2):263-283. https://doi.org/10.1016/j. pbb.2006.09.021

32. Smith ER, DeCoster J (2000) Dual-process models in social and cognitive psychology: conceptual integration and links to underlying memory systems. Pers Soc Psychol Rev 4(2):108-131

33. Fazio RH, Olson MA (2003) Implicit measures in social cognition. research: their meaning and use. Annu Rev Psychol 
54:297-327. https://doi.org/10.1146/annurev.psych.54.101601. 145225

34. Teachman BA, Clerkin EM, Cunningham WA et al (2019) Implicit cognition and psychopathology: looking back and looking forward. Annu Rev Clin Psychol 15:123-148. https://doi. org/10.1146/annurev-clinpsy-050718-095718

35. Roefs A, Huijding J, Smulders FTY et al (2011) Implicit measures of association in psychopathology research. Psychol Bull 137(1):149-193. https://doi.org/10.1037/a0021729

36. Gawronski B, de Houwer J (2014) Implicit measures in social and personality psychology. In: Reis HT, Judd CM (eds) Handbook of research methods in social and personality psychology. Cambridge University Press, pp 283-310

37. Greenwald AG, McGhee DE, Schwartz JLK (1998) Measuring individual differences in implicit cognition: the implicit association test. J Pers Soc Psychol 74(6): 1464

38. Payne BK, Cheng CM, Govorun O et al (2005) An inkblot for attitudes: affect misattribution as implicit measurement. J Pers Soc Psychol 89(3):277-293. https://doi.org/10.1037/0022-3514. 89.3.277

39. Clerkin EM, Teachman BA (2010) Training implicit social anxiety associations: an experimental intervention. J Anxiety Disord 24(3):300-308

40. Bar-Anan Y, Nosek BA (2014) A comparative investigation of seven indirect attitude measures. Behav Res Methods 46(3):668-688

41. Payne K, Lundberg K (2014) The affect misattribution procedure: ten years of evidence on reliability, validity, and mechanisms. Soc Pers Psychol Compass 8(12):672-686. https://doi.org/10.1111/spc3. 12148

42. Payne BK, Hall DL, Cameron CD et al (2010) A process model of affect misattribution. Pers Soc Psychol Bull 36(10):1397-1408. https://doi.org/10.1177/0146167210383440

43. Schreiber F, Neng JMB, Heimlich C et al (2014) Implicit affective evaluation bias in hypochondriasis: findings from the affect misattribution procedure. J Anxiety Disord 28(7):671-678. https://doi. org/10.1016/j.janxdis.2014.07.004

44. Spring VL, Bulik CM (2014) Implicit and explicit affect toward food and weight stimuli in anorexia nervosa. Eat Behav 15(1):91-94. https://doi.org/10.1016/j.eatbeh.2013.10.017

45. Schreiber F, Bohn C, Aderka IM et al (2012) Discrepancies between implicit and explicit self-esteem among adolescents with social anxiety disorder. J Behav Ther Exp Psychiatry 43(4):1074-1081

46. Wong J, Morrison AS, Heimberg RG et al (2014) Implicit associations in social anxiety disorder: the effects of comorbid depression. J Anxiety Disord 28(6):537-546. https://doi.org/10.1016/j.janxdis. 2014.05.008

47. Harb GC, Heimberg RG, Fresco DM et al (2002) The psychometric properties of the Interpersonal Sensitivity Measure in social anxiety disorder. Behav Res Ther 40(8):961-979

48. Petermann F, Petermann U (2008) HAWIK-iv. Kindheit und Entwicklung 17(2):71-75

49. Petermann, F (2012) Wechsler Adult Intelligence Scale-Fourth Edition (WAIS-IV) (German Version), Pearson Assessment, Frankfurt am Main

50. Arbeitsgruppe Deutsche Child Behavior Checklist (1998a) Fragebogen für Jugendliche: deutsche Bearbeitung des Youth Self-Report (YSR) der Child Behavior Checklist. Einführung und Anleitung zur Handauswertung. 2. Ed., Arbeitsgruppe Kinder-, Jugend und Familiendiagnostik (KJFD), Cologne

51. Arbeitsgruppe Deutsche child behavior checklist (1998b) Elternfragebogen über das Verhalten von Kindern und Jugendlichen; deutsche Bearbeitung der Child Behavior Checklist (CBCL/4-18). Einführung und Anleitung zur Handauswertung. Einführung und Anleitung mit deutschen Normen. Arbeitsgruppe Kinder-, Jugenddiagnostik (KJFD), Cologne
52. Bradley MM, Lang PJ (1994) Measuring emotion: the self-assessment manikin and the semantic differential. J Behav Ther Exp Psychiatry 25(1):49-59

53. Müller B, Winter B, Schürkens A et al (2004) Validierung und Normierung von kindgerechten, standardisierten Bildmotiven aus dem International Affective Picture System. Untersuchung an einer deutschen Feldstichprobe von Kindern im Alter zwischen sechs und zwölf Jahren (Rating and standardisation of pictures from the International Affective Picture System appropriated for children. Examination of children in a field sample aged six to twelve years). Z Kinder Jugendpsychiatr Psychother 32(4):235-243. https://doi. org/10.1024/1422-4917.32.4.235

54. Greenwald AG, Nosek BA, Banaji MR (2003) Understanding and using the implicit association test: I. An improved scoring algorithm. J Pers Soc Psychol 85(2):197

55. Langner O, Dotsch R, Bijlstra G et al (2010) Presentation and validation of the Radboud Faces Database. Cogn Emot 24(8):1377-1388

56. Döpfner M, Görtz-Dorten A, Lehmkuhl G (2008) DISYPS-II: Diagnostik-System für psychische Störungen nach ICD-10 und DSM-IV für Kinder und Jugendliche-II: Manual. H. Huber

57. Dose C, Görtz-Dorten A, Breuer D, Goletz H, Döpfner M (2014) Die Erfassung von Ängsten im Urteil von Kindern/Jugendlichen und ihren Eltern. Eine Analyse mit den Angstfragebögen aus dem Diagnostik-System (DISYPS-II). Diagnostica 61(4):197-209

58. Melfsen SS, Warnke A, Florin I (2001) Sozialphobie und-angstinventar für Kinder: SPAIK. Hogrefe, Manual

59. Melfsen S, Florin I, Warnke A (2001) Das Sozialphobie und -angstinventar für Kinder (SPAIK). Hogrefe, Goettingen

60. Beidel DC, Turner SM, Hamlin K, Morris TL (2000) The Social Phobia and Anxiety Inventory for Children (SPAI-C): external and discriminative validity. Behav Ther 31(1):75-87

61. Beck AT, Steer RA, Brown GA, Hautzinger M, Keller F, Kühner C (2006) Beck-Depressionsinventar (BDI-II). Pearson, Frankfurt am Main.

62. Kühner C, Bürger C, Keller F et al (2007) Reliabilität und validität des revidierten beck-depressionsinventars (BDI-II). Nervenarzt 78(6):651-656

63. Dozois DJA, Dobson KS, Ahnberg JL (1998) A psychometric evaluation of the Beck Depression Inventory-II. Psychol Assess 10(2):83

64. Rao PA, Beidel DC, Turner SM et al (2007) Social anxiety disorder in childhood and adolescence: descriptive psychopathology. Behav Res Ther 45(6): 1181-1191

65. Alfano CA, Beidel DC, Turner SM (2006) Cognitive correlates of social phobia among children and adolescents. J Abnorm Child Psychol 34(2):189-201. https://doi.org/10.1007/s10802-005-9012-9

66. Downey G, Feldman SI (1996) Implications of rejection sensitivity for intimate relationships. J Pers Soc Psychol 70(6):1327

67. Liu RT, Kraines MA, Massing-Schaffer M et al (2014) Rejection sensitivity and depression: Mediation by stress generation. Psychiatry 77(1):86-97

68. Chango JM, McElhaney KB, Allen JP et al (2012) Relational stressors and depressive symptoms in late adolescence: rejection sensitivity as a vulnerability. J Abnorm Child Psychol 40(3):369-379

69. Pearson KA, Watkins ER, Mullan EG (2010) Submissive interpersonal style mediates the effect of brooding on future depressive symptoms. Behav Res Ther 48(10):966-973

70. Tops M, Riese H, Oldehinkel AJ et al (2008) Rejection sensitivity relates to hypocortisolism and depressed mood state in young women. Psychoneuroendocrinology 33(5):551-559

71. Stuijfzand S, Creswell C, Field AP et al (2018) Research review: is anxiety associated with negative interpretations of ambiguity in children and adolescents? A systematic review and meta-analysis. J Child Psychol Psychiatry 59(11):1127-1142. https://doi.org/10. 1111/jcpp. 12822

72. Holmes EA, Lang TJ, Shah DM (2009) Developing interpretation bias modification as a "cognitive vaccine" for depressed mood: 
Imagining positive events makes you feel better than thinking about them verbally. J Abnorm Psychol 118(1):76-88. https://doi.org/10. 1037/a0012590

73. Beard C, Amir N (2008) A multi-session interpretation modification program: changes in interpretation and social anxiety symptoms. Behav Res Ther 46(10):1135-1141. https://doi.org/10.1016/j.brat. 2008.05.012

74. Verpaalen IAM, Bijsterbosch G, Mobach L et al (2019) Validating the Radboud faces database from a child's perspective. Cogn Emot 33(8):1531-1547. https://doi.org/10.1080/02699931.2019.1577220
75. Durand K, Gallay M, Seigneuric A et al (2007) The development of facial emotion recognition: the role of configural information. J Exp Child Psychol 97(1):14-27. https://doi.org/10.1016/j.jecp.2006.12. 001

76. Muris P, Merckelbach H, Damsma E (2000) Threat perception bias in nonreferred, socially anxious children. J Clin Child Psychol 29(3):348-359. https://doi.org/10.1207/S15374424JCCP2903_6

77. Koyuncu A, Ertekin E, Binbay Z et al (2014) The clinical impact of mood disorder comorbidity on social anxiety disorder. Compr Psychiatry 55(2):363-369 